\title{
Existência e Unicidade de Solução para um sistema de Equações de Maxwell aplicado a um condutor perfeito
}

\author{
$\underline{\text { Marcos T. Alves, }}$ \\ PPGMA, UFPR \\ 81531-980, Curitiba, PR \\ E-mail: mtmarcos@gmail.com, \\ Marcio V. Ferreira \\ UFSM - Departamento de Matemática \\ 97105-900, Santa Maria, RS \\ E-mail: v.f.marcio@gmail.com.
}

Resumo: Investigaremos questões relacionadas a existência e unicidade de solução para um sistema de equações de Maxwell num meio condutor perfeito. Esse estudo será baseado na caracterização dos espaços funcionais de origem eletromagnética e na aplicação da teoria de semigrupos de operadores lineares.

Palavras-chave: Espaços Funcionais em Eletromagnetismo. Operador de Maxwell. Equações de Maxwell. Semigrupos de operadores lineares.

Em 1873, James Clerk Maxwell (físico e matemático britânico) fundou a teoria moderna do eletromagnetismo com a publicação de sua obra Treatise on Eletricity and Magnetism, reunindo um conjunto de equações que atualmente levam seu nome.

Estas equações são o suporte matemático para os fenômenos eletromagnéticos, estabelecendo equações diferenciais que relacionam os campos elétrico e magnético e suas respectivas induções. Representaremos por $E, H, D$ e $B$, os campos elétrico, magnético e as induções elétrica e magnética, respectivamente. Nas aplicações tratadas neste trabalho, consideramos os campos que variam no tempo. Desse modo,

$$
E=E(x, t), H=H(x, t), D=D(x, t) \text { e } B=B(x, t)
$$

são funções vetoriais que dependem da posição espacial $x=\left(x_{1}, x_{2}, x_{3}\right) \in \mathbb{R}^{3}$ e do tempo $t \in \mathbb{R}$, $t \geq 0$.

Os campos apresentados anteriormente são gerados por dois tipos de fontes: cargas elétricas estáticas e fluxos de cargas elétricas variáveis chamadas correntes. A distribuição de cargas é dada por uma função escalar $\rho$ que representa a densidade de carga elétrica, enquanto que as correntes são descritas por uma função vetorial de densidade de corrente $J$.

A relação existente entre os campos $E, H, D$ e $B$ e as fontes $\rho$ e $J$ é sintetizada por um conjunto de equações que, em geral, são aplicadas a uma região do espaço $\mathbb{R}^{3}$ ocupada por um campo eletromagnético. Estas equações, denominadas equações de Maxwell, em sua forma diferencial são as seguintes:

$$
\begin{array}{r}
\frac{\partial D}{\partial t}-\nabla \times H=-J, \\
\frac{\partial B}{\partial t}+\nabla \times E=0, \\
\nabla \cdot D=\rho, \\
\nabla \cdot B=0 .
\end{array}
$$


Descrevemos agora cada uma das equações listadas acima.

A equação (1) é conhecida como Lei de Ampère-Maxwell. Esta lei coincide com a Lei de Ampère salvo pela adição do termo $\frac{\partial D}{\partial t}$ introduzido por Maxwell.

A equação (2) é chamada Lei de Faraday. Ela fornece o efeito da variação da indução magnética no campo elétrico.

A equação (3), conhecida como Lei de Gauss, estabelece que o fluxo de indução elétrica através de uma superfície fechada é dado pela carga líquida dentro da superfície, o que significa que as linhas de cargas elétricas começam e terminam com cargas elétricas.

A Lei de Gauss para o magnetismo aparece na equação (4) e afirma que o fluxo da indução magnética ao longo de qualquer superfície fechada é nulo.

A partir das equações (1) e (3), relacionamos as densidades $\rho$ e $J$ através da equação

$$
\frac{\partial \rho}{\partial t}+\nabla \cdot J=0
$$

conhecida como equação da conservação da eletricidade.

Dada a complexidade do sistema (1)-(4), juntaremos a estas leis universais certas leis constitutivas que incorporam, de forma suficientemente simples, interações eventualmente complexas e que traduzam características particulares dos materiais sem comprometer, dentro de determinados limites, a adequação entre o modelo e a realidade física.

Uma das leis constitutivas aqui consideradas é a Lei de Ohm, que destaca uma relação de proporcionalidade entre o campo elétrico $E$ e a densidade de corrente $J$, ou seja,

$$
J=\sigma E,
$$

onde $\sigma$ representa a condutividade do material. Além dessa, dado nosso interesse por modelos de Maxwell aplicados em condutores perfeitos ${ }^{1}$, tomamos as leis de magnetização e polarização do meio da forma

$$
D=\varepsilon E \text { e } B=\mu H
$$

em que $\varepsilon$ representa a capacidade indutiva e $\mu$ representa a permeabilidade magnética do meio.

De posse das equações (5) e (6) e considerando $J=0, \rho=0$ (campo eletromagnético no vácuo com ausência de cargas), reescrevemos o sistema (1)-(4) como segue

$$
\begin{aligned}
\frac{\partial(\varepsilon E)}{\partial t}-\nabla \times H & =0, \\
\frac{\partial(\mu H)}{\partial t}+\nabla \times E & =0, \\
\nabla \cdot(\varepsilon E) & =0, \\
\nabla \cdot(\mu H) & =0 .
\end{aligned}
$$

Neste trabalho, adotaremos $\varepsilon=\mu=1$. Desse modo, abordaremos o seguinte sistema de equações de Maxwell

$$
\begin{array}{r}
\frac{\partial E}{\partial t}-\nabla \times H=0, \\
\frac{\partial H}{\partial t}+\nabla \times E=0, \\
\nabla \cdot E=0, \\
\nabla \cdot H=0 .
\end{array}
$$

No instante $t=0$, iremos supor que o campo elétrico $E(x, 0)=E_{0}(x)$ e o campo magnético $H(x, 0)=H_{0}(x)$ sejam conhecidos e satisfaçam as relações:

$$
\nabla \cdot E_{0}=0 \text { e } \nabla \cdot H_{0}=0 .
$$

\footnotetext{
${ }^{1} \mathrm{Um}$ condutor perfeito é um meio fictício tal que $\sigma \rightarrow+\infty$. No interior de um condutor perfeito, o campo é nulo. Metais são materiais que aproximam-se deste conceito.
} 
Acrescentamos às condições iniciais, as condições de fronteira do meio em estudo. Para esse propósito, seja $\Omega$ uma região do espaço $\mathbb{R}^{3}$ representando um condutor perfeito e $\Gamma$ sua fronteira. Fisicamente, diante dessas condições, faz sentido considerarmos as seguintes condições de fronteira

$$
E \times\left.\eta\right|_{\Gamma}=0 \text { e }\left.H \cdot \eta\right|_{\Gamma}=0,
$$

em que $\eta(x)=\left(\eta_{1}(x), \eta_{2}(x), \eta_{3}(x)\right)$ denota o vetor normal unitário a $x \in \Gamma$ exterior a $\Omega$.

Sob essas hipóteses, um de nossos objetivos nessa apresentação será discutir questões relacionadas a existência e unicidade de solução para o sistema de equações de Maxwell (7)-(10) sujeito às condições iniciais em (11) e às condições de fronteira em (12).

Os trabalhos assentes neste modelo linear usam um conjunto de técnicas que passam pela formulação fraca das equações a resolver, permitindo encontrar soluções com menor regularidade do que aquela que é exigível a soluções das equações dos modelos encontrados, referido vulgarmente por formulação forte do problema.

Observamos que nos problemas de eletromagnetismo intervêm essencialmente os operadores diferenciais vetoriais de primeira ordem: divergente e rotacional. Assim, torna-se natural adotarmos os espaços das funções de $\left[L^{2}(\Omega)\right]^{3}$ cujo divergente ou rotacional são funções de $L^{2}(\Omega)$ e $\left[L^{2}(\Omega)\right]^{3}$, respectivamente, como quadro funcional para o estudo desses fenômenos. Estes espaços são designados por $H(\operatorname{div}, \Omega)$ e $H(r o t, \Omega)$.

Com este espírito, o foco deste trabalho consiste na caracterização destes espaços e em suas aplicações em modelos eletromagnéticos específicos. Dessa forma, elaboramos o roteiro que passamos a descrever.

Inicialmente, visando um tratamento matemático para os problemas de origem eletromagnética considerados, trataremos dos espaços $H(\operatorname{div}, \Omega)$ e $H($ rot,$\Omega)$ do ponto de vista de suas propriedades topológicas. Enaltecemos neste momento a caracterização dos traços das funções de $H($ div,$\Omega)$ e $H($ rot,$\Omega)$; passo fundamental para dispormos de uma fórmula de integração por partes. Em seguida, definiremos os subespaços $H(\operatorname{div} 0, \Omega)$ e $H_{0}(\operatorname{div} 0, \Omega)$ de $\left[L^{2}(\Omega)\right]^{3}$. Estudaremos propriedades de densidade nestes espaços essenciais para as aplicações. Os principais resultados referentes ao estudo desses espaços funcionais são descritos abaixo:

Teorema 1 (do Traço para $H(d i v, \Omega)$ ) Seja $\Omega$ um conjunto aberto do $\mathbb{R}^{3}$ com fronteira $\Gamma$ limitada e Lipschitz. Então

(i) $[\mathcal{D}(\bar{\Omega})]^{3}$ é denso em $H($ div,$\Omega)$;

(ii) A aplicação traço, $\gamma_{\eta}:\left.v \mapsto v \cdot \eta\right|_{\Gamma}$ definida em $[D(\bar{\Omega})]^{3}$, estende-se por continuidade a uma aplicação linear, contínua e sobrejetiva, ainda denotada por $\gamma_{\eta}$, de $H($ div,$\Omega)$ sobre $H^{-1 / 2}(\Gamma)$ (aqui $\eta(x)$ denota a normal exterior unitária em $x \in \Gamma$ );

(iii) É válida a seguinte igualdade:

$\int_{\Omega}(\nabla \cdot v) \varphi d x+\int_{\Omega} v \cdot(\nabla \varphi) d x=<\gamma_{\eta}(v), \varphi>_{H^{-1 / 2}(\Gamma), H^{1 / 2}(\Gamma)}, \forall v \in H(d i v, \Omega), \varphi \in H^{1}(\Omega)$,

conhecida como "fórmula generalizada de Green" para o espaço $H($ div, $\Omega)$;

(iv) O núcleo $\operatorname{ker}\left(\gamma_{\eta}\right)$ da aplicação $\gamma_{\eta}$ é o espaço $H_{0}(\operatorname{div}, \Omega)$.

O próximo fato ilustra uma condição suficiente para que funções de $H(r o t, \Omega)$ pertençam ao espaço $H_{0}($ rot,$\Omega)$.

Teorema 2 Seja $\Omega$ um conjunto aberto do $\mathbb{R}^{3}$ com fronteira $\Gamma$ limitada e Lipschitz. Se tivermos $u \in H($ rot,$\Omega)$ tal que

$$
(u, \nabla \times \varphi)-(\nabla \times u, \varphi)=0, \forall \varphi \in[\mathcal{D}(\bar{\Omega})]^{3},
$$

então $u \in H_{0}($ rot,$\Omega)$. 
Com auxílio do teorema anterior, caracterizamos o traço de funções de $H(r o t, \Omega)$.

Teorema 3 (do Traço para $H(r o t, \Omega)$ ) Seja $\Omega$ um conjunto aberto do $\mathbb{R}^{3}$ com fronteira $\Gamma$ limitada e Lipschitz. Então

(i) $[\mathcal{D}(\bar{\Omega})]^{3}$ é denso em $H($ rot,$\Omega)$;

(ii) A aplicação traço, $\gamma_{\tau}: v \mapsto v \times\left.\eta\right|_{\Gamma}$ definida em $[D(\bar{\Omega})]^{3}$, estende-se por continuidade a uma aplicação linear e contínua, ainda denotada por $\gamma_{\tau}$, de $H(r o t, \Omega)$ em $\left[H^{-1 / 2}(\Gamma)\right]^{3}$ (aqui $\eta(x)$ denota a normal exterior unitária em $x \in \Gamma$ );

(iii) Para todo $v \in H($ rot,$\Omega)$ e para todo $\varphi \in\left[H^{1}(\Omega)\right]^{3}$, vale a seguinte igualdade:

$$
\int_{\Omega} v \cdot(\nabla \times \varphi) d x-\int_{\Omega}(\nabla \times v) \cdot \varphi d x=<\gamma_{\eta}(v), \varphi>_{\left[H^{-1 / 2}(\Gamma)\right]^{3},\left[H^{1 / 2}(\Gamma)\right]^{3}}
$$

conhecida como "fórmula generalizada de Green" para o espaço $H($ rot,$\Omega)$;

(iv) $O$ núcleo $\operatorname{ker}\left(\gamma_{\tau}\right)$ da aplicação $\gamma_{\tau}$ é o espaço $H_{0}($ rot,$\Omega)$.

Via teorema de traço em $H(r o t, \Omega)$, obtemos a seguinte "fórmula de integração":

$$
\int_{\Omega} w \cdot(\nabla \times v) d x=\int_{\Omega}(\nabla \times w) \cdot v d x, \text { para todos } w \in H_{0}(\text { rot }, \Omega) \text { e } v \in H(\text { rot }, \Omega) .
$$

O desfecho deste tratamento matemático para a teoria do Eletromagnetismo ocorre com algumas aplicações dos espaços funcionais abordados anteriormente. Como primeira aplicação, estudamos o sistema de equações de Maxwell com uma condição de fronteira dada por $\eta \times E=0$. Para este fim, definiremos o operador de Maxwell $\mathcal{A}$ e caracterizaremos o operador $\mathcal{A}^{*}$ (adjunto de $\mathcal{A})$. Em nossa última aplicação, restringiremos este operador ao subespaço

$$
\begin{aligned}
\mathcal{H} & =\left\{(E, H) \in X ; \nabla \cdot E=0, \nabla \cdot H=0,\left.\eta \cdot H\right|_{\Gamma}=0\right\} \\
& =H(\operatorname{div} 0, \Omega) \times H_{0}(\operatorname{div} 0, \Omega),
\end{aligned}
$$

obtendo assim o operador $\mathcal{A}_{\mathcal{H}}$. Com auxílio da teoria de semigrupo de operadores lineares, encerraremos a apresentação provando o seguinte teorema de existência e unicidade de solução para um sistema de equações de Maxwell num meio condutor perfeito:

Teorema 4 Dado $\left(E_{0}, H_{0}\right) \in D\left(\mathcal{A}_{\mathcal{H}}\right)$, existe uma única solução

$$
E, H \in \mathcal{C}\left([0,+\infty), D\left(\mathcal{A}_{\mathcal{H}}\right)\right) \cap \mathcal{C}^{1}([0,+\infty), \mathcal{H})
$$

do problema $(7)-(12)$.

\section{Referências}

[1] Alves, M. T. Espaços Funcionais e Operadores Lineares em Eletromagnetismo. 2012. Dissertação (Mestrado em Matemática) - Universidade Federal de Santa Maria, Santa Maria, 2012.

[2] Dautray, R.; Lions, J. L. Mathematical Analysis and Numerical Methods for Science and Technology, Spectral Theory and Applications. Berlin: Spring-Verlag, 1990, v. 3.

[3] Ferreira, M. V.; Perla Menzala, G. Uniform Stabilization of an electromagnetic-elasticity problem in exterior domain, Discrete and Continuous Dynamical Systems, v. 18, n. 4, p. 719-746, 2007. 
[4] Ferreira, M. V. Ondas Elásticas e Electromagnéticas em Domínios Exteriores: Propriedades Assintóticas. 2005. Tese (Doutorado em Matemática) - Universidade Federal do Rio de Janeiro, Rio de Janeiro, 2005.

[5] Girault, V.; Raviart, P. A., Finit Element Methods for Navier-Stokes Equations. Berlin: Springer-Verlag, Berlin, 1986.

[6] Medeiros, L. A.; Miranda, M. M. Espaços de Sobolev: iniciação aos problemas elíticos não homogêneos. Rio de Janeiro: Instituto de Matemática - UFRJ, 2000.

[7] Monk, P. Finite Element Methods for Maxwell's Equations. New York: Oxford University Press, 2003.

[8] Nicaise, S. Exact Boundary Controllability of Maxwell's Equations in Heterogeneous Media and an Application to an Inverse Source Problem. SIAM Journal on Control Optimization, v. 38 , n. 4 , p. $1145-1170,2000$.

[9] Pazy, A. Semigroups of Linear Operators and Applications to Partial Differential Equations. New York: Springer-Verlag, 1983.

[10] Phung, K. D. Contrôle et Stabilisation D'Ondes Électromagnétiques. ESAIM: Control, Optimisation and Calculus of Variations, v. 5, p. 87-137, 2000.

[11] Yin, H. On Maxwell's equations in an electromagnetic field with the temperature effect. SIAM Journal on Mathematical Analysis, v. 29, n. 3, p. 637-651, 1998. 